\title{
The Visual Literacy Dimension of Community Communication: Illustration Preferences of a Rural Community in South Africa
}

\author{
Rolf J. Gaede
}

\begin{abstract}
The chapter discusses the role of visual literacy in a community communication setting and specifically focuses on the process of identifying pictorial communication preferences in the Thibela community of the rural Qwa Qwa region in South Africa. The aim was to collect empirical information on the basis of which easily comprehensible and appropriately illustrated nutrition education material about growing and using soya beans, produced in a visual style acceptable to the community, could be developed. The data collection and analysis procedures were guided by core concepts from the visual literacy literature, including the notion of visual representational latitude (VRL) as described by Pauwels, as well as the contractual axis of semiosis, which forms part of Johansen's semiotic pyramid model. The data collection approach involved a ten to fifteen minutes long, voluntary and anonymous session in the respondent's home language during which a field worker completed a questionnaire in the presence of the participant $(\mathrm{n}=75)$. The questionnaire was designed with a view to accommodate low-literate participants and comprised items relating to pictorial referent recognition, varying degrees of visual abstraction, as well as a range of illustrative styles, including pictograms, clip art and hand-drawn images. The outcome was that a clear consensus emerged among the participants about the type of pictorial signs that should be included, and the manner in which they should be used. The questionnaire data also illustrate the main strengths and weaknesses associated with the different illustrative styles according to the views and opinions of the respondents, and underscore the widely accepted view that inappropriate visual images have the potential to generate an extensive range of unintended meanings. On the strength of the data collected, a visually illustrated nutrition education calendar was produced and then distributed free of charge in the community.
\end{abstract}

Key Words: Visual literacy, community communication, pictorial semiotics, visual representational latitude, discourse ethics.

$* * * * *$

\section{Introduction}

The present chapter deals with the on-going process of developing soy education materials meant for a low-literate target group in the Thibela community of the mountainous Qwa-Qwa region in South Africa. The chapter focuses on the visual communication dimension of these training materials. The training materials form part of the Qwa-Qwa integrated nutrition education programme, which is 
primarily funded by the South Africa Netherlands Research Programme on Alternatives in Development (SANPAD). The overarching aim of the programme is to explore to what extent food production and nutrition education training programmes, complemented by the implementation of soy home gardening initiatives and innovative household soy processing equipment as interventions, impact on household food security, as well as positive changes in dietary intake and food procurement patterns. ${ }^{1}$

Visual images play an important role in this type of setting, especially in cases where the verbal literacy level of the target group is low. For the purpose of the chapter, the 1995 UNESCO definition of illiteracy is used, ${ }^{2}$ according to which an illiterate person is unable to read or write with adequate understanding a simple sentence about everyday life. Based on the four levels of verbal literacy outlined by Wagner $^{3}$ (non-literate, low-literate, moderate-literate and high-literate), the Thibela community may be described as low-literate. That is not to say that each and every member of the community is necessarily unable to read a text with understanding or unable to write a short text in a locally used language on the one hand, but able to recognise several words in everyday contexts, or perform simple tasks such as supplying a signature, on the other hand. The term 'low-literate' only describes the state of affairs in the community as a whole, and is based on the number of years of formal schooling received. In the 212 Qwa-Qwa households who participated in the programme thus far (i.e., a total of 1018 people, or 212 households with a mean of 4.8 people per household), $14.7 \%$ reported not having received any formal education at all, 30.4\% received primary school education (i.e., 1-7 years of school attendance), and $54.9 \%$ reported secondary school education or higher.

The aim of the study was to collect empirical information during a dialogue albeit a highly structured dialogue - between the producer of the soy education training material and the members of the target community about the type of visual signs that should be included, as well as the manner in which they should be used. On the basis of this empirical information, easily comprehensible and appropriately illustrated nutrition education material about growing and using soya beans, produced in a visual style acceptable to the community, could then be produced.

The remainder of the chapter comprises two parts. In the first part the theoretical underpinnings of the data collection and analysis procedures are briefly outlined. The second part describes the process of designing and implementing a questionnaire suitable for a low-literate target community, as well as the data that the questionnaire yielded.

\section{Theoretical Underpinnings}

The most important concept that guided the study design is visual representational latitude (VRL) as described by Pauwels. ${ }^{4}$ VRL forms part of a visual literacy framework with the emphasis on visual representational practices in science. Specifically, VRL refers to 'coping with controlled and uncontrolled 
variations in the depicted and the depiction, ${ }^{5}$ and thus links closely with the notions of vagueness and indeterminacy. Pauwels writes that:

Visual representational latitude, therefore, is not just a producer's (or sender's) problem; that is, it is not just a matter of deciding how to express variation, of choosing the right level of iconicity or abstraction for a specific purpose. It is also a user's (or receiver's) problem: what kind of variation is to be expected in the real world, and which elements in this particular representation are 'motivated' by a perceived reality, and which others are due to specific, intentional or unintentional choices of the producer, limitations of the medium or larger production context? ${ }^{6}$

In other words, a visual illustration with a narrow VRL is associated with a relatively stable meaning and a low degree of vagueness or indeterminacy. In contrast, an image with a wide VRL tends to give rise to an extensive range of possible interpretations and may be described as essentially 'open' in nature. In the case of nutrition education materials, it should be self-evident that the aim is to ensure that the majority of the images used as part of the training materials have a sufficiently narrow VRL in order to avoid needlessly confusing the target group. In addition to finding ways of reducing the indeterminacy of the pictorial signs used in this type of setting, the visual illustrations should also be acceptable to the target community in a wider sense. Stated differently, the visual illustrations should be associated with a high degree of universalisation, to use a term from Habermas' work in the area of discourse ethics. ${ }^{7}$ The discourse principle of universalisation requires the acceptance (Zustimmung) of the communicative norms at play by all involved, implying both an agreement (Einverständnis) and a contract (Vereinbarung). Habermas writes that:

Only those norms can claim validity that could meet with the acceptance of all concerned in a practical discourse. ... A norm is valid when the foreseeable consequences and side effects of its general observance for the interests and value-orientations of each individual could be jointly accepted by all concerned without coercion. ${ }^{8}$

In the context of the present chapter, this means that the quality of the agreement reached (on the contractual axis of semiosis in Johansen's semiotic pyramid model ${ }^{9}$ ) between the producer of the visual illustrations (or the semiotic self) and the target community (or the semiotic other) about the visual content of the training materials is crucial for their success in practice. 


\section{Design and Methods}

Data collection involved voluntary, anonymous participation based on informed consent during which a community member and a field worker held a ten to fifteen minutes long conversation in the home language of the participant in the local community hall. The data collection took place on a day selected by the community leaders and the event was announced by sending all participating households SMS messages, followed up by reminders on the community radio station. During the conversation between the field worker $(\mathrm{n}=4)$ and the respondent (a total of 75 community members participated), the field worker completed a short questionnaire.

The questionnaire covered basic demographic information about the participant (age, home language, gender), as well as several images which varied with regard to the extent of visual abstraction, as well as the illustration style. On the one end of the spectrum were highly abstract black and white pictograms, such as those used in airports. In the middle of the spectrum were plain line (or outline-only) drawings and clip art images as found in standard software programs. At the end of the spectrum the questionnaire also included shaded line drawings with a high degree of realism (or a high degree of correspondence between the referent and the depiction).

The questionnaire was designed to accommodate low-literate respondents and to avoid a situation where the ability of the participant to verbally articulate complex pictorial meanings was essential. This was achieved by restricting the preformulated questions to the following: (a) What does this image show? and (b) The illustration style of the two images is different. Which one of the two do you prefer? In both cases the participant was invited to make general comments or to explain further. The field workers were trained to remain as neutral as possible during data collection, regardless of the responses encountered.

In one particular case, for example, the field worker showed the participating community member a clip art image of a soy bean and asked 'What does this image show?' The respondent answered 'It's a picture of the moon.' The field worker neither nodded nor shook his head but simply recorded the answer in the space provided. As he was writing, the respondent added 'It's a picture of the moon with a banana on the moon' (due to the curved black line on soy beans). The field worker added the new information to the response, remained as neutral and factual as possible, and accepted what the respondent had said.

\section{Results}

In short, the outcome of the field work was that a clear consensus emerged among the participants ( $n=75)$ about the type of pictorial signs that should be included, and the manner in which they should be used. As far as the VLR of the images in the questionnaire is concerned, the responses were assessed by comparing them to an envisaged meaning of the visual illustration in question. For 
example, in the case of a clip art image of a soy bean, drawn from existing training materials used elsewhere, a reply along the lines of 'a bean' or 'soy' were recorded as a correct or envisaged answer, in contrast to replies such as 'a football' or 'the moon,' which were entered on the database as incorrect, or an interpretation at variance with the intended meaning. In the case of this particular example, 9.3\% $(n=7)$ of the respondents supplied the envisaged answer, indicating an unacceptably wide VLR, in contrast to other images in the questionnaire where more than $90 \%$ of the respondents gave the envisaged answer. Seen as a whole, the responses to the questionnaire item 'What does this image show?' suggest that images with a high degree of visual abstraction were associated with low referent recognition in the target group. Clip art images were very poorly understood throughout.

As far as the target community's illustration preferences are concerned, the respondents consistently indicated that they favour sparingly shaded outline drawings with a high degree of pictorial realism. In one of the questionnaire items, for instance, the respondents were shown two images side by side illustrating the washing of hands under a tap and asked the question 'The illustration style of the two images is different. Which one of the two do you prefer?' The first image was rendered in a black and white pictogram-type illustration style with minimal detail, whereas the second illustration contained considerably more image detail (such as the thread at the end of the tap where a hosepipe can be attached) and was drawn and shaded with a high degree of realism. As $86.7 \%(n=65)$ of the respondents chose the second illustration, and $13.3 \%(n=10)$ opted for the first image, there can be no doubt were the community's pictorial illustration preference lies, especially when the accompanying comments were taken into account as well.

\section{Conclusion}

Based on the above described questionnaire results, a soy education calendar was produced according to the community's visual illustration preferences and then distributed free of charge as part of the Qwa-Qwa integrated nutrition education programme. A special effort was made to ensure that the overall visualverbal balance of the final product was appropriate for a low-literate target group, and that all images associated with a wide VLR were edited out. Flowing from a comment made by one of the respondents, the illustrated borders of the final calendar were based on photographs taken of the patterns decorating some of the community's exterior house walls, known as Litema, ${ }^{10}$ as well as examples of these on display at the Basotho Cultural Village near the town of Phuthaditjhaba. In this way, a link was created between elements of the established visual culture of the community and the nutrition education calendar.

Having reached the end of the project, a question which lingers is: How structured should the dialogue between the producer of the visual illustrations and the target community ideally be? In the case of this particular study, the degree of 
structuration was very high, and this produced clear results. However, good researchers always ask whether the outcome of a particular investigation would have been substantially different if the process of data collection had been approached differently. Obtaining an answer to this question requires extending the dialog between the researcher and the target community beyond a discussion about visual illustration preferences and the manner in which visual signs should be employed, to include an exchange of opinions and ideas about the data collection procedure itself, or 'a dialogue about the dialogue.'

\section{Notes}

${ }^{1}$ Wilna Oldewage-Theron, 'Introduction', in Centre of Sustainable Livelihoods Symposium Proceedings, ed. Wilna Oldewage-Theron (Vanderbilbark: Vaal University of Technology, 2011), 3.

${ }^{2}$ UNESCO, Compendium of Statistics on Illiteracy, No. 35 (Paris: United Nations Educational, Scientific and Cultural Organization., 1995), 4.

${ }^{3}$ Daniel A. Wagner. Literacy, Culture and Development: Becoming Literate in Morocco (Cambridge: Cambridge University Press, 1993), 260.

4 Luc Pauwels. 'Scientific Discourse and Visual Representational Literacy: Elements and Dimensions of an Integrated Theoretical Framework', in Visual Literacy and Development, eds. Robert Griffin, Scott Chandler and Belle Cowden (Loretto, PA: International Visual Literacy Association, 2005), 9.

${ }^{5}$ Ibid.

${ }^{6}$ Ibid.

${ }^{7}$ Jürgen Habermas, 'On the Relation between the Nation, the Rule of Law and Democracy', in The Inclusion of the Other. Studies in Political Theory, eds. Ciara Cronin and Pable de Greiff (Cambridge: MIT Press, 1998), 141.

${ }^{8}$ Ibid.

9 Jørgen Dines Johansen, Dialogic Semiotics: An Essay on Signs and Meaning, (Bloomington: Indiana University Press, 1993).

${ }^{10}$ Carina M Beyer, Litema - Mural Masterpiece (Bloemfontein: Central University of Technology, Free State, 2008).

\section{Bibliography}

Beyer, Carina M. Litema - Mural Masterpiece. Bloemfontein: Central University of Technology, Free State, 2008. 
Habermas, Jürgen. 'On the Relation between the Nation, the Rule of Law and Democracy'. In The Inclusion of the Other. Studies in Political Theory, edited by Ciara Cronin, and Pable de Greiff, 129-153. Cambridge: MIT Press, 1998.

Johansen, Jørgen Dines. Dialogic Semiotics: An Essay on Signs and Meaning. Bloomington: Indiana University Press, 1993.

Oldewage-Theron, Wilna. 'Introduction'. In Centre of Sustainable Livelihoods Symposium Proceeding, edited by Wilna Oldewage-Theron, 3-5. Vanderbilbark: Vaal University of Technology, 2011.

Pauwels, Luc. 'Scientific Discourse and Visual Representational Literacy: Elements and Dimensions of an Integrated Theoretical Framework'. In Visual Literacy and Development, edited by Robert Griffin, Scott Chandler, and Belle Cowden, 1-10, Loretto, PA: International Visual Literacy Association, 2005.

UNESCO. Compendium of Statistics on Illiteracy, No. 35. Paris: United Nations Educational, Scientific and Cultural Organization, 1995.

Rolf J. Gaede is an Associate Professor at the Vaal University of Technology in South Africa. His main research interests are visual methods and visual semiotics. 

Exploring Visual Literacy Inside, Outside and through the Frame 


\title{
At the Interface
}

\author{
Series Editors \\ Dr Robert Fisher \\ Dr Daniel Riha
}

\section{Advisory Board}

Dr Alejandro Cervantes-Carson

Professor Margaret Chatterjee

Dr Wayne Cristaudo

Mira Crouch

Dr Phil Fitzsimmons

Professor Asa Kasher

Owen Kelly
Dr Peter Mario Kreuter

Martin McGoldrick

Revd Stephen Morris

Professor John Parry

Paul Reynolds

Professor Peter Twohig

Professor S Ram Vemuri

Revd Dr Kenneth Wilson, O.B.E

A At the Interface research and publications project. http://www.inter-disciplinary.net/at-the-interface/

The Education Hub

'Visual Literacies'

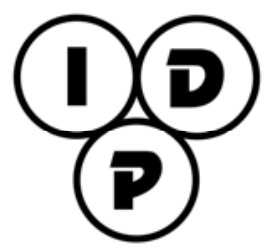




\section{Exploring Visual Literacy Inside, Outside and through the Frame}

Edited by

Aundreta Conner Farris and Frieda Pattenden

Inter-Disciplinary Press

Oxford, United Kingdom 
(C) Inter-Disciplinary Press 2012

http://www.inter-disciplinary.net/publishing/id-press/

The Inter-Disciplinary Press is part of Inter-Disciplinary.Net - a global network for research and publishing. The Inter-Disciplinary Press aims to promote and encourage the kind of work which is collaborative, innovative, imaginative, and which provides an exemplar for inter-disciplinary and multi-disciplinary publishing.

All rights reserved. No part of this publication may be reproduced, stored in a retrieval system, or transmitted in any form or by any means without the prior permission of Inter-Disciplinary Press.

Inter-Disciplinary Press, Priory House, 149B Wroslyn Road, Freeland, Oxfordshire. OX29 8HR, United Kingdom.

$+44(0) 1993882087$

ISBN: 978-1-84888-112-9

First published in the United Kingdom in eBook format in 2012. First Edition. 


\section{Table of Contents}

\section{Introduction}

Aundreta Conner Farris and Frieda Pattenden

\section{Part 1 Inside, Outside and through the Frame}

The Frame and the Window: Rhetoric Value in the Visual Field

Alexandra Ai Quintas

\section{Part 2 Framing Praxis}

National Myth and National Identity: The Visual

'Framing' of Ambivalence

Phil Fitzsimmons and Edie Lanphar

The Visual Literacy Dimension of Community Communication: Illustration Preferences of a Rural Community in South Africa

Rolf J. Gaede

Drawing on Art Practice: Rethinking Visual Processes and Pedagogies

Tracey Bowen

Reading the Image: Visual Literacy and the Films of Jean Rouch

Rayma Watkinson

Readers/Viewers: Popular Culture and Visual Literacy in Shteyngart, McEwan, Chabon and Egan

Ben De Bruyn

\section{Part 3 Through the Scope of Industry}

The Creation of New Values in Japanese Texts through the Use of Multimodal Communication 
A Study on Visual Literacy: Similarities between Visual Strategies in Portuguese Concrete Poems of the 70s and Contemporary Ads

Elsa Simões Lucas Freitas

Contemporary Visual Literacies: Advertising, Politics and Architecture

Graham Cairns

\section{Part 4 Visual Form}

Two Lessons in Reading the Picture: John Ruskin vs.

Roger Fry

Aleksandra Piasecka

Rhetoric of Ruins: Camilo José Vergara, Walter

Benjamin and the Politics of Urban Photography

Christopher Carter

\section{Part 5 Display}

Foundations of Visual Literacy: Historic Preservation and Image Management

Margot Note

Images for Deification: Visual Literacy in Marguerite Porete's Mirror of Simple Souls

Pablo García Acosta

The Spiritual Diagram: Theology and Didactics in Saint John of the Cross

Anna Serra Zamora

Displaying Difference: Signifying Conventions in the Presentation of Indigenous Australian Art

Lisa Chandler

Investigating the Vestibule of the Text: Paratext Beverley Croker 


\section{Part 6 Constructing and Obstructing Gender}

The Camera’s Eye and I: Image-ing the First Person

Singular

Wendy C. Turgeon

Visually Reading The Sopranos: 'You are All White

Professional Males between 25 and 45'

Frieda Pattenden

Female Enclosure, Surveillance, and Prurient Expectations of the Contemporary Audience: Visualising the Medieval in Newby's Film Anchoress

Rebecca D. Flynn

\section{Part 7 Illustrating the Frame}

The Rest is Silence: Visual Literacy and Shifting

Significations in Nicki Greenberg's Hamlet:

Staged on the Page

LJ Maher

Me and My Shadow

John Pratt

Identity and Visual Culture in David Wiesner's

The Three Pigs and Art \& Max

Lesley D. Clement 